advising other departments on the development of their services and how to manage individual problems. The subtle denigration of which you speak is a consequence of the present structure in which rheumatologists are often asked to take over the care of another specialist's patients when they feel they can do no more. The process of rehabilitation should start from the time the patient is first seen and requires an attitude of mind by all members of the clinical team.

You know that I am an active supporter of the development of the specialty of rehabilitation. I believe that the best way forward is the emergence of the specialty of rehabilitation in its own right rather than as an appendage to rheur:atology.

MaLCOLM I. V. JAYSON Professor of Rheumatology, Rheumatic Diseases Centre, University of Manchester

\section{Reactive arthritis associated with campylobacter enteritis}

Sir, It was with interest that we read of the recent survey by Gumpel et al. ${ }^{1}$ of patients in the Harrow Health District infected with campylobacter species during 1978. We are currently analysing the results of a similar survey of a single milk borne outbreak of Campylobacter jejuni originating from one dairy in a rural district south of Aberdeen. ${ }^{2}$

One hundred and twenty-six patients under the care of 2 general practitioners, who attend all patients in the relevant area, were infected with Campylobacter jejuni in the months of January and February of 1979 and were traced through the records of the Regional Laboratory, City Hospital, Aberdeen, where all samples were sent. One hundred and thirteen patients continue to reside in the area and their general practice records have been examined. Two patients have subsequently attended hospital clinics. One of these, a male aged 41 , presented within 1 month of his gastroenteritis with left-sided sciatica to a local orthopaedic colleague. Six months previously he had had a right-sided sciatica. His notes indicate that he had evidence of nerve root irritation, and his symptoms spontaneously resolved. The second patient was an 8-year-old girl who presented 2 months after initial infection with intermittent fever, muscle aching, and joint pains. She continues to have episodes of diarrhoea. She was seen by a paediatric colleague, who could find no clinical abnormality of the joints. Her ESR was normal. There was no evidence from repeated stool cultures of reinfection within the family.

A third patient, a 34-year-old male, did not attend hospital, but presented to his general practitioner about 1 month after infection with a right olecranon bursitis. No other patients' records indicate any medical attention for musculoskeletal complaints within 3 months of their bowel infection.

To complete the survey symptomatic patients are to be contacted and their clinical history reviewed, personally, On the basis of the results of this survey so far the frequency of inflammatory joint disease would appear to be considerably less in this single outbreak of Campylobacter jejuni enteritis in the community than in the 33 hospitalised patients seen by Gumpel et al. It is of interest that they were unable to identify any examples of arthritis in patients who had not been hospitalised for their infection. This, together with the results from our own survey, suggests that the severity of the gastroenteritis may be of importance in addition to genetic factors in determining the development of a subsequent reactive arthritis. Alternatively, the sample from our study may be less biased than the Harrow series. Our study is of a single outbreak during which close cooperation between the Regional Laboratory and the general practitioners ensured the collection of faecal samples from all symptomatic patients presenting together with contacts, thus ensuring as complete a sample as possible. It is well recognised that hospitalised patients are not representative of a population sample with the same disease.

C. J. EASTMOND*
T. M. S. REID $\dagger$
J. A. N. RENNIE*
${ }^{*}$ Department of Rheumatology* and
$\dagger$ Regional Laboratory, City Hospital,
Urquhart Road, Aberdeen AB9 SAU.

\section{References}

1 Gumpel J M, Martin C, Sanderson P J. Reactive arthritis associated with campylobacter enteritis. Ann Rheum Dis 1981; 40: 64-5.

2 Porter I A, Reid T M S. A milk-borne outbreak of campylobacter infection. J Hyg 1980; 84: 415-9.

\section{CRVO and scleroderma}

Sir, In their interesting article Littlejohn et al..$^{1}$ described a case of central retinal vein occlusion (CRVO) in a patient with progressive systemic sclerosis (PSS). According to the authors this might have been a manifestation of sclerodermatous vascular disease at the level of the fundus oculi. While this hypothesis is doubtless correct, we would like to point out that occlusion of the central retinal vein is rare in PSS patients and, in general, in patients with connective tissue disorders. By means of simple ophthalmoscopy we failed to find this lesion in a nonselected series of 71 patients with PSS. Grade III or IV hypertensive retinopathy was present in 2 of these patients with sclerodermatous malignant hypertension. We found abnormalities of the fundus vessels in another 24 , but 10 of these patients were above 60 years of age. Other eye lesions found in the above series of 71 patients were: mild choroiditis (2), nodular episcleritis (1), mild keratitis (2), markedly reduced tear secretion (4), and induration of the lower lid (48). CRVO was found only in 2 out of 31 cases of undefined connective tissue disease (one of the 2 with probable polyarteritis nodosa). On the other hand CRVO was not found in patients with systemic lupus erythematosus (31 cases), dermatomyositis and polymyositis (13 cases), undifferentiated 